\title{
TITLE: Thoracic versus abdominal approach to correct diaphragmatic eventration in
}

\section{children}

Alisha Gupta ${ }^{1}$, Martin Sidler ${ }^{1,2}$, Daan van Poll ${ }^{1}$, Nikita Patel ${ }^{1}$, Simon Eaton ${ }^{3}$, Nagarajan Muthialu ${ }^{3}$, Paolo De Coppi ${ }^{1,2}$ on behalf of the Diaphragmatic Eventration Group

${ }^{1}$ Department of Specialist Neonatal and Paediatric Surgery, Great Ormond Street Hospital for Children NHS trust, London, UK

${ }^{2}$ Stem Cells and Regenerative Medicine section, Developmental Biology and Cancer Programme, Institute of Child Health, University College London, London WC1N 1EH, UK

${ }^{3}$ Department of Cardiothoracic Surgery, Great Ormond Street Hospital for Children NHS Trust

Diaphragmatic eventration Group consists of Kate Cross, Stefano Giuliani, Simon Blackburn, Martin Kostolny, Martin Elliott, Joseph Curry, Great Ormond Street Hospital for Children NHS Trust

\section{Corresponding author}

Prof. Paolo De Coppi

Great Ormond Street Hospital for Children NHS trust

London, United Kingdom

WC1N 3JH

Phone: 02074059200 (Ext. 5906)

paolo.decoppi@gosh.nhs.uk 
TITLE: Thoracic versus abdominal approach to correct diaphragmatic eventration in children

\section{Abstract}

\section{Background}

Plication of diaphragm (DP) for eventration (DE) can be done using thoracic or abdominal approaches. The purpose of our study was to compare outcomes between these approaches.

\section{Methods}

Retrospective records of children $<16$ years who underwent DP (single-centre, 20042018) were recorded and analysed. Data are reported as median (range).

\section{Results}

Eighty-nine cases were identified in thoracic (Congenital=5, Acquired=84) and 13 (Congenital=10, Acquired=3) in abdominal group aged $5.88(0.36-184.44$ ) and 10.0 (0.12-181.8) months. Improvement in diaphragm level post-DP was significantly higher in abdominal [2(0-4)] than chest [1.5(0-5)] group $(p=0.04)$. On Cox regression analysis, there was no difference in time to extubation $(p=0.2)$ or time to feed $(p=0.18)$ between the 2 groups. Patients operated transthoracically left intensive care unit after a significantly longer time $(p=0.04)$. All 16 recurrences were in the chest group although this was not statistically significant due to skewed patient numbers $(p=0.19)$.

\section{Conclusion}

This is one of the largest reports on outcomes of children undergoing DP for DE. There was no significant difference in recurrence rate, even though all recurrences in our series (15.7\%) were in the acquired cases operated using a thoracic approach. 


\section{Keywords}

Diaphragmatic eventration, plication, thoracotomy, thoracoscopy, laparotomy, laparoscopy

\section{Level of evidence}

Level III (Treatment retrospective comparative study) 
Diaphragmatic eventration (DE) is not very common among paediatric patients. However, successful diaphragmatic plication (DP) can be life changing for the affected children. DE can either be 'congenital' as a result of defective embryological development of the diaphragmatic muscle or 'acquired' secondary to phrenic nerve damage as a result of birth trauma or mediastinal surgery [1,2]. Congenital DE is rare with an incidence of 0.02-0.07 per 1000 births [3]. Phrenic nerve palsy has been observed in $0.3-12.8 \%$ of children undergoing cardiac surgery [4,5]. As the consequences of DE from either aetiology are similar, they are usually discussed and reported together.

Surgical repair of DE in children essentially involves plicating the redundant central portion of the diaphragm. Different routes have been employed for surgical access, beginning with a laparotomy in 1927 followed by open transthoracic, thoracoscopic and laparoscopic approaches $[6,7]$.

While proponents of a thoracic approach claim the advantage of better visualisation of the phrenic nerve and inherent circumvention of the peritoneal cavity, proponents of an abdominal approach proclaim a liberal working space, avoidance of single lung ventilation and clear visualisation of adjacent structures, especially bowel [8-10]. In the minimally invasive surgery (MIS) era, similar plea have been advocated for thoracoscopic and laparoscopic repairs in addition to the common tenets of quicker recovery, smaller incisions and decreased pain [11].

Personal experiences of various surgeons with each of these approaches has been reported in literature, but there is no defined 'best practice' to date. We report here our experience with the use of both routes for DP and compare outcomes, especially recurrence, between transthoracic and transabdominal approaches.

\section{Material and methods}

\subsection{Patients}


Following institutional audit approval (Ref No. 2509), case records of patients (<16 years age) who underwent surgical repair (DP) of DE at a single tertiary referral centre between January 2004 and December 2018 were retrospectively reviewed. Patient characteristics, diagnosis, management and outcomes were recorded and analysed. Outcomes included improvement in the level of diaphragm, as assessed by the difference in number of rib levels on a plain chest $X$-ray before and after the operation; time to extubation; length of intensive care unit (ICU) stay; time to feed and recurrence.

\subsection{Statistical analysis}

We divided our patients into 2 groups - those operated via a thoracic or an abdominal approach. Data were analysed applying standard statistical tests using software IBM SPSS Version 25 (SPSS Inc., Chicago, IL, USA). Patient characteristics were compared using Mann-Whitney $U$ test for continuous variables. Fisher's exact test was used for dichotomous variables. Outcomes between the two groups were analysed using multivariate Cox-regression and the log-rank test for univariate analysis. P value of $\leq 0.05$ was considered significant. 


\section{Results}

A total of 102 patients (65 boys, 37 girls) underwent DP for DE. Eighty-nine patients (87\%) underwent transthoracic (Open=86, MIS=3) and 13 (13\%) transabdominal (Open=4, MIS=9) plication of the diaphragm (Table 1). The groups were comparable with regards to gender distribution, age, weight at surgery and laterality. Most children had an acquired DE (87/102) secondary to phrenic nerve injury related to cardiac $(n=80)$ or other mediastinal surgery $(n=7)$ and were operated using chest approach in $84 / 87$ (97\%). On the other hand, a significantly higher proportion of children had congenital DE in the abdominal group.

Median improvement in the level of diaphragm was significantly greater in the transabdominal as compared to the transthoracic group (2 rib levels vs. 1.5 rib levels respectively; $p=0.04$ ) (Figure 1 ).

Time to extubation was compared between chest and abdominal approaches by Cox regression analysis, with age at diagnosis, open/MIS and congenital/acquired as covariates and this was not significant (hazard ratio $=0.54[0.21-1.40] p=0.2$ ). Patients operated by the chest approach left ICU after a significantly longer time (hazard ratio $=0.34[0.12-0.97] p=0.04)$. There was no difference between time to feed between the 2 groups (hazard ratio=1.80 [0.76-4.25] $\mathrm{p}=0.18$ ).

There were 16 short term recurrences in the chest group and none in the abdominal group but this was non-significant (log-rank test, hazard ratio=0.32 [0.06-1.68], $p=0.19)$. Of 16 recurrences in the transthoracic group, 13 underwent a redo transthoracic operation, whereas 3 underwent transabdominal procedure for their second repair. There were 17 post-operative complications in addition to the recurrences, these are listed in Table 2; there was one post-operative mortality from necrotising enterocolitis. 


\section{Discussion}

The diaphragm is perhaps the only organ in the human body that is so strategically placed so as to allow access from two completely different directions. Paediatric surgeons, over the years, have taken advantage of this fact and successfully approached it through the chest as well as the abdomen, beginning with open surgical procedures and moving on to minimally invasive techniques as technology has advanced. Majority of the literature on paediatric DE repair describes personal or single institutional experiences using one approach or the other [12-24] with limited number of multicentric studies $[25,26]$. Pros and cons of each of these approaches have been clearly defined by various adult and paediatric studies but there is no consensus regarding 'best practice'. Whether outcomes between the two approaches are different or not was the driver for us to review our own experience using both approaches.

The primary outcome of clinical interest is the recurrence rate after surgery. We found all our recurrences in the transthoracic group only (16/102), although a comparison with the abdominal group is limited by the shorter follow-up. It is notable that the recurrences can be grouped into 'early' (<1 year) or 'late'; although the abdominal group appears to have no early recurrences, we do not yet know whether there may be late recurrences. On aetiological segregation, 15/16 recurrences were in children with 'acquired' and 1/16 in 'congenital' DE. The majority of the studies previously published in literature have reported nil recurrence rates except for two, one describing outcomes after the thoracoscopic approach (25\% recurrence) and one reporting on laparoscopic repair (46\% recurrence) $[21,25]$. In the first study, the thoracoscopic technique involved use of an endostapler to resect the redundant diaphragm in one of the patients with recurrence [25]. This method is not standard practice and the authors have given a word of caution against it. The other patient experiencing a recurrence underwent a thoracoscopic DP both as primary and redo procedure. The second study reported recurrences in 6 of 13 children after laparoscopic repair and all underwent redo laparoscopic repair with no further recurrences reported [21]. 
Difference in the level of the highest point of diaphragm can be calculated using a pre and post surgical chest radiograph. This could be an objective indicator of 'effectiveness' of the procedure, although post-operative requirement of respiratory support defines 'success' clinically. We found a higher degree of improvement in the diaphragmatic level after the abdominal as compared to the chest approach. This might be related to better visualisation of the entire diaphragm, including costophrenic recesses, especially when laparoscopic artificial pneumoperitoneum balloons it out like a dome. This allows the surgeon to take stitches as peripherally as needed to make the diaphragm taught, keeping the bowel safely retracted. On the contrary, this visualisation of the entire periphery is compromised in a thoracotomy, especially if done after previous cardiothoracic surgery, which can lead to dense adhesions. Additionally, in thoracoscopic operations, an artificial pneumothorax may keep the diaphragm relatively flat due to the increased intrathoracic pressure, thereby making it difficult to gauge the effectiveness of the repair intra-operatively. Previous studies have assessed improvement in level of diaphragm post plication, but none has compared this parameter between different approaches [14,24].

Time to extubation is an indirect evidence of improvement in respiratory function. We found a longer time to extubation and intensive care requirement in patients operated using a transthoracic versus a transabdominal approach. This may be attributed to post-operative pain and splinting of diaphragm after a thoracotomy; or single lung ventilation, hypercarbia and artificial pneumothorax causing impaired venous return after a thoracoscopic procedure causing haemodynamic and respiratory compromise $[27,28]$. Previously published studies have reported successful median time to extubation between 0-2 days after transthoracic plication, erring on the lesser side for MIS as compared to open technique [15-18,22]. In a review of 18 cases of acquired DE operated laparoscopically, median time to extubation was 0 (0-2) days [14]. However, no difference was noted in another study comparing thoracoscopic and laparoscopic repairs [25]. 
Another important surgical outcome measure is the time to commence feeds. We found comparable time to commence feeds in the chest and abdominal groups. An abdominal operation with handling of bowel can cause a brief period of post-operative ileus that is clearly avoided in a thoracic surgery. However, with laparoscopic procedures, bowel handling is usually less than in an open abdominal operation. Median time to feeds in a previously reported study was 1.6 days (1-4) in both groups and this was not significantly different [25].

Our study has several limitations. Our own experience is a retrospective review with limited data on surgical decision-making and limited follow-up, especially in the abdominal group. We found no significant difference in recurrence rates when comparing approach (chest or abdomen), aetiology (congenital or acquired) or technique (open or minimally invasive). We did, however, note a higher recurrence rate in trans-thoracically repaired, acquired DEs plicated using open technique, yet asymmetric patient distribution between groups in all 3 categories (approach, aetiology and technique) limits the validity of the analysis. Which of the three is the single most important predictor of outcome remains to be proven using a prospectively conducted study.

\section{Conclusion}

Our experience of 102 patients who underwent DP for DE is the largest paediatric series reported to date. We found a higher recurrence rate with transthoracic as compared to transabdominal repair. Further evidence is required to define the optimal approach.

\section{Acknowledgement}

P.D.C. and M.S. are supported by National Institute for Health Research (NIHR-RP2014-04-046). All research at Great Ormond Street Hospital NHS Foundation Trust and UCL Great Ormond Street Institute of Child Health is made possible by the NIHR Great Ormond Street Hospital Biomedical Research Centre. The views expressed are 
those of the author (s) and not necessarily those of the NHS, the NIHR or the Department of Health.

\section{References}

[1] Deslauriers J: Eventration of the diaphragm. Chest Surg Clin N Am 1998; 8:315-330.

[2] Riley EA: Idiopathic diaphragmatic paralysis; a report of eight cases. Am J Med 1962; 32:404-416.

[3] Jurcak-Zaleski S, Comstock CH, Kirk JS: Eventration of the diaphragm. Prenatal diagnosis. J Ultrasound Med 1990; 9:351-354.

[4] de Leeuw M, Williams JM, Freedom RM, et al: Impact of diaphragmatic paralysis after cardiothoracic surgery in children. J Thorac Cardiovasc Surg 1999; 118:510-517. [5] van Onna IE, Metz R, Jekel L, et al: Post cardiac surgery phrenic nerve palsy: value of plication and potential for recovery. Eur J Cardiothorac Surg 1998; 14:179-184.

[6] Podgaetz E, Diaz I, Andrade RS: To Sink the Lifted: Selection, Technique, and Result of Laparoscopic Diaphragmatic Plication for Paralysis or Eventration. Thorac Cardiovasc Surg 2016; 64:631-640.

[7] Cilley RE, Coran AG: Eventration of the diaphragm. In: Smith R, Editor. Pediatric surgery, 1995. p. 168-75.

[8] Tönz M, von Segesser LK, Mihaljevic T, et al: Clinical implications of phrenic nerve injury after pediatric cardiac surgery. J Pediatr Surg 1996; 31:1265-1267.

[9] Ribet M, Linder JL: Plication of the diaphragm for unilateral eventration or paralysis. Eur J Cardiothorac Surg 1992; 6:357-360.

[10] Tiryaki T, Livanelioğlu Z, Atayurt H: Eventration of the diaphragm. Asian J Surg 2006; 29:8-10.

[11] Iwanaka $T$, Arai $M$, Ito $M$, et al: Laparoscopic surgery in neonates and infants weighing less than $5 \mathrm{~kg}$. Pediatr Int 2000; 42:608-612.

[12] Alshorbagy A, Mubarak Y: Open Transthoracic Plication of the Diaphragm for Unilateral Diaphragmatic Eventration in Infants and Children. Korean J Thorac Cardiovasc Surg 2015; 48:307-310.

[13] Ghribi A, Bouden A, Braiki M, et al: Diaphragmatic eventration in children. Tunis Med 2015; 93:76-78.

[14] Cao GQ, Tang ST, Aubdoollah TH, et al: Laparoscopic Diaphragmatic 
Hemiplication in Children with Acquired Diaphragmatic Eventration After Congenital Heart Surgery. J Laparoendosc Adv Surg Tech A 2015; 25:852-857.

[15] Fujishiro J, Ishimaru T, Sugiyama M, et al: Thoracoscopic plication for diaphragmatic eventration after surgery for congenital heart disease in children. $J$ Laparoendosc Adv Surg Tech A 2015; 25:348-351.

[16] Hines MH: Video-assisted diaphragm plication in children. Ann Thorac Surg 2003; 76:234-236.

[17] Baker CJ, Boulom V, Reemtsen BL, et al: Hemidiaphragm plication after repair of congenital heart defects in children: quantitative return of diaphragm function over time. J Thorac Cardiovasc Surg 2008; 135:56-61.

[18] Becmeur F, Talon I, Schaarschmidt K, et al: Thoracoscopic diaphragmatic eventration repair in children: about 10 cases. J Pediatr Surg 2005; 40:1712-1715. [19] Lao VV, Lao OB, Abdessalam SF: Laparoscopic transperitoneal repair of pediatric diaphragm eventration using an endostapler device. J Laparoendosc Adv Surg Tech A $2013 ; 23: 808-813$.

[20] Joho-Arreola AL, Bauersfeld U, Stauffer UG, et al: Incidence and treatment of diaphragmatic paralysis after cardiac surgery in children. Eur J Cardiothorac Surg $2005 ; 27: 53-57$.

[21] Borruto FA, Ferreira CG, Kaselas C, et al: Thoracoscopic treatment of congenital diaphragmatic eventration in children: lessons learned after 15 years of experience. Eur J Pediatr Surg 2014; 24:328-331.

[22] Snyder CW, Walford NE, Danielson PD, et al: A simple thoracoscopic plication technique for diaphragmatic eventration in neonates and infants: technical details and initial results. Pediatr Surg Int 2014; 30:1013-1016.

[23] Wu S, Zang N, Zhu J, et al: Congenital diaphragmatic eventration in children: 12 years' experience with 177 cases in a single institution. J Pediatr Surg 2015; 50:10881092.

[24] Hu J, Wu Y, Wang J, et al: Thoracoscopic and laparoscopic plication of the hemidiaphragm is effective in the management of diaphragmatic eventration. Pediatr Surg Int 2014; 30:19-24. 
[25] Miyano G, Yamoto M, Kaneshiro M, et al: Diaphragmatic eventration in children: laparoscopy versus thoracoscopic plication. J Laparoendosc Adv Surg Tech A 2015; 25:331-334.

[26] Yazici M, Karaca I, Arikan A, et al: Congenital eventration of the diaphragm in children: 25 years' experience in three pediatric surgery centers. Eur J Pediatr Surg 2003; 13:298-301.

[27] Hill RC, Jones DR, Vance RA, et al: Selective lung ventilation during thoracoscopy: effects of insufflation on hemodynamics. Ann Thorac Surg 1996; 61:945-948.

[28] Jones DR, Graeber GM, Tanguilig GG, et al: Effects of insufflation on hemodynamics during thoracoscopy. Ann Thorac Surg 1993; 55:1379-1382. 


\section{$\underline{\text { Table legends }}$}

Table 1. Patient characteristics of transthoracic and transabdominal diaphragmatic plication groups

Table 2. Post-operative complications as per Clavien-Dindo classification 


\section{Figure legends}

Figure 1. Median preoperative as well as postoperative rib levels of the diaphragmatic dome were lower by 2 rib levels ( $8^{\text {th }}$ vs. $6^{\text {th }}$ rib) or 1.5 rib levels ( 9.5 vs. $8^{\text {th }} \mathrm{rib}$ ) in the chest group compared to the abdominal group, respectively $(p<0.001$ both comparisons). However, median improvement in diaphragm level was greater after abdominal approach compared to the chest approach [2 (0-4) rib levels vs. 1.5 (0-5) rib levels; $p=0.04]$.

Figure 2. Although all 16 recurrences were in the chest group, but this was not statistically significant due to skewed patient numbers in both the groups (Hazard ratio $=0.32[0.06-1.68], p=0.19)$. 
Table 1. Patient characteristics and outcomes between transthoracic and transabdominal diaphragmatic plication groups

\begin{tabular}{|l|c|c|c|}
\hline & Chest & Abdomen & P value \\
\hline Number of patients (Total, $\mathrm{n}=102)$ & 89 & 13 & \\
\hline Sex [M/F] & $57 / 32$ & $8 / 5$ & $>0.99$ \\
\hline Age at surgery [months] $\diamond$ & $5.88(0.36-184.4)$ & $10.0(0.1-181.8)$ & 0.601 \\
\hline Weight at surgery [kg] $\diamond$ & $5.86(1.3-41.7)$ & $8.45(3-50)$ & 0.282 \\
\hline Laterality [Right/Left/Bilateral] & $45 / 43 / 1$ & $6 / 7 / 0$ & 0.803 \\
\hline Aetiology [Congenital/Acquired] & $5 / 84$ & $10 / 3$ & $<0.001$ \\
\hline Surgical technique [Open/minimally invasive] & $86 / 3$ & $4 / 9$ & $<0.001$ \\
\hline Follow-up [months] $\diamond$ & $3.8(0-137)$ & $3.2(0-26.2)$ & 0.372 \\
\hline
\end{tabular}

$\diamond$ Data reported as median (range)

Table 2. Post-operative complications as per Clavien-Dindo classification

\begin{tabular}{|l|c|c|}
\hline Complications & n & Clavien-Dindo grade \\
\hline Chest group & 1 & III-b \\
\hline Haemothorax & 4 & II (n=2) \\
\hline Lung collapse/consolidation & 3 & V (n=1) \\
\hline Necrotising enterocolitis & 1 & I \\
\hline Pneumothorax & 2 & I \\
\hline Pyopneumothorax & 3 & I \\
\hline Chylothorax & 1 & III-b \\
\hline Cardiac arrhythmia & 2 & I \\
\hline Abdominal group & 1 & \\
\hline Adhesive bowel obstruction & & \\
\hline Lung consolidation & & \\
\hline
\end{tabular}


Figure 1

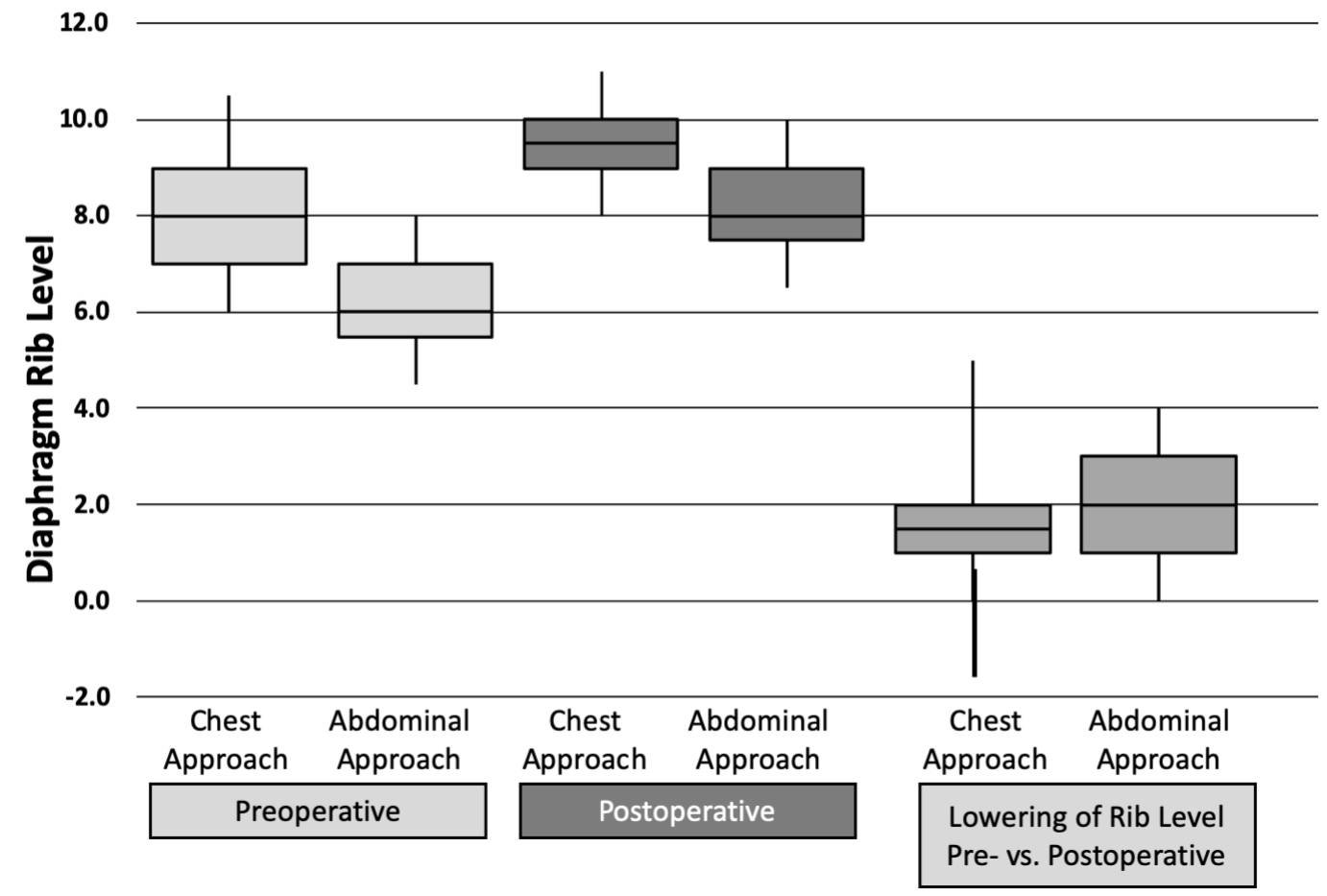

Figure 2

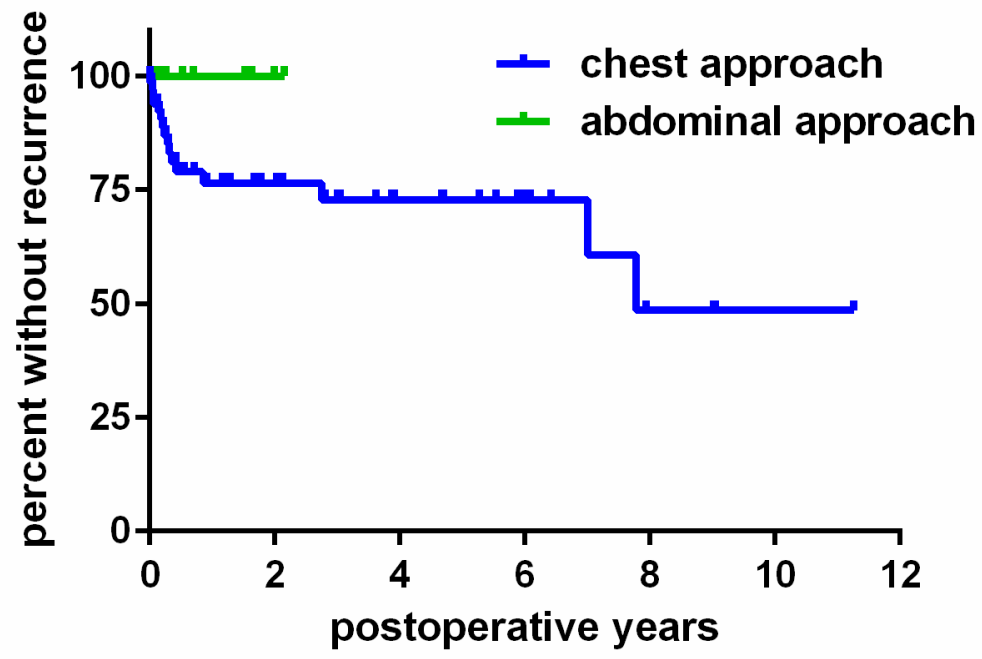

